\title{
ON THE RADII OF STARLIKENESS AND CONVEXITY OF CERTAIN CLASSES OF REGULAR FUNCTIONS
}

\author{
PRAN NATH CHICHRA \\ (Received 23 October 1969; revised 27 February 1970) \\ (Communicated by E. Strzelecki)
}

\section{Introduction}

Let $R_{n}$ denote the class of functions $f(z)=z+a_{n} z^{n}+\cdots(n \geqq 2)$ which are regular in the open disc $|z|<1$ (hereafter called $E$ ) and satisfy

$$
\operatorname{Re}\left(\frac{f(z)}{z}\right)>0
$$

for all $z$ in $E . R_{n}$ is a subclass of the class of close-to-star functions in $E[9$, p. 61]. MacGregor showed that the radius of univalence and starlikeness of $R_{n}$ is $\left[1-n+\left(n^{2}-2 n+2\right)^{\frac{1}{2}}\right]^{1 /(n-1)}$, see [4,5]. The radius of convexity of $R=R_{2}$ is $r_{0}=0.179 \cdots$, where $r_{0}$ is the smallest positive root of the equation $1-5 r-3 r^{2}-$ $r^{3}=0$, see [8].

In this paper we consider a subclass $R_{n}(\alpha)$ of the class $R_{n}$, the members of $R_{n}(\alpha)$ being those members of $R_{n}$ which satisfy

$$
\left|\frac{f(z)}{z}-\alpha\right|<\alpha \quad\left(\alpha>\frac{1}{2}\right),
$$

for all $z$ in $E$. The main purpose of this paper is to find the radius of convexity of $R(\alpha)=R_{2}(\alpha)$. To obtain the result in more general form we further assume that $f(z)$ is $k$-fold symmetric, that is, it has power series expansion of the form

$$
f(z)=z+\sum_{m=1}^{\infty} a_{m k+1} z^{m k+1} .
$$

We also obtain the radius of univalence and starlikeness of $R_{n}(\alpha)$. Corresponding result for the class $R(1)$ is known to be $\frac{1}{2}$, see [6]. By making $\alpha$ tend to infinity in the above results for the class $R_{n}(\alpha)$, we can obtain the corresponding results for the class $R_{n}$.

For the above classes the identity function $z$ plays a key role. It would naturally be interesting to see how the radii of univalence and convexity vary when the identity function is replaced by some other function $g(z)$ such that $g(0)=0$; 
that is, to investigate similar problems for the class of functions $f(z)$ which are regular in $E$ and satisfy $f(0)=0, f^{\prime}(0)=1$, and

$$
\left|\frac{f(z)}{g(z)}-\alpha\right|<\alpha \quad\left(\alpha>\frac{1}{2}\right),
$$

for all $z$ in $E$. We take $g(z)=z+a z^{2}(|a| \leqq 1)$ and find the radius of univalence and starlikeness of the above class. We also find the radius of convexity of the above class when $\alpha=1$, or $\infty$. It is found that these radii decrease monotonically as $|a|$ increases from 0 to 1 .

The estimates used to obtain the above results are further used to obtain the radius of univalence and starlikeness of a subclass of the class of typically real functions.

\section{2}

We shall need the following lemmas.

Throughout this paper $P(z)$ denotes a function which is regular in $E$ and satisfies $P(0)=1, \operatorname{Re} P(z)>0$ for all $z$ in $E$.

LEMMA 1. If $P(z)$ has a power series expansion of the form

$$
P(z)=1+c_{n} z^{n}+c_{n+1} z^{n+1}+\cdots(n \geqq 1),
$$

then for $|z|=r, 0 \leqq r<1$,

$$
\left|P^{\prime}(z)\right| \leqq \frac{2 n r^{n-1}}{\left(1-r^{2 n}\right)} \operatorname{Re} P(z),
$$

and

$$
\left|\frac{P^{\prime}(z)}{P(z)+\mu}\right| \leqq \frac{2 n r^{n-1}}{\left(1-r^{n}\right)\left[\left(1+r^{n}\right)+\left(1-r^{n}\right) \cdot \operatorname{Re}(\mu)\right]},
$$

where $\mu$ is any complex number with $\operatorname{Re}(\mu) \geqq 0$.

Corresponding results for $n=1$ and $\mu=0$ are due to Libera [3] and MacGregor [5].

Proof. Let

$$
f(z)=\frac{1-P(z)}{1+P(z)}
$$

Then $f(z)$ is regular in $E$ and satisfies $|f(z)|<1$ for $z$ in $E$ [7, p. 169]. Also $f(z)$ has a zero at $z=0$ of order at least $n$ and hence by Schwarz's lemma $|f(z)| \leqq|z|^{n}$. For such functions we have [1]

$$
\left|f^{\prime}(z)\right| \leqq \frac{n r^{n-1}}{\left(1-r^{2 n}\right)}\left(1-|f(z)|^{2}\right), \quad|z|=r<1 .
$$


Substituting for $f(z)$ from (2.4) we obtain (2.2).

Further

$$
\begin{aligned}
\left|\frac{P^{\prime}(z)}{P(z)+\mu}\right| & \leqq \frac{\left|P^{\prime}(z)\right|}{\operatorname{Re}(P(z))+\operatorname{Re}(\mu)} \\
& =\frac{\left|P^{\prime}(z)\right|}{\operatorname{Re}(P(z))} \frac{1}{1+(\operatorname{Re}(\mu) / \operatorname{Re}(P(z)))},
\end{aligned}
$$

which in view of (2.2) and the inequality [4]

$$
\operatorname{Re}(P(z)) \leqq \frac{1+r^{n}}{1-r^{n}}
$$

yields (2.3).

It is easy to show that equality holds in (2.2) and (2.3) for $\mu \geqq 0$ only for functions $P(z)=\left(1-\varepsilon z^{n}\right) /\left(1+\varepsilon z^{n}\right)$ where $|\varepsilon|=1$ and for appropriate values of $z$.

Lemma 2. If $P(z)$ has power series expansion of the form (2.1), then for $|z|=r<\mid$ and $\mu \geqq 0$,

$$
\left|\frac{1}{P(z)+\mu}-\frac{(\mu+1)-(\mu-1) r^{2 n}}{(\mu+1)^{2}-(\mu-1)^{2} r^{2 n}}\right| \leqq \frac{2 r^{n}}{\left[(\mu+1)^{2}-(\mu-1)^{2} r^{2 n}\right]} .
$$

Proof. Let $\psi(z)=1 /(P(z)+\mu)$. Substituting for $P(z)$ in terms of $\psi(z)$ in (2.4) and using the fact that $|f(z)| \leqq|z|^{n}$, we obtain for $|z|=r<1$,

$$
\left|\frac{\psi(z)-(1 /(\mu+1))}{\psi(z)-(1 /(\mu-1))}\right| \leqq\left|\frac{\mu-1}{\mu+1}\right| r^{n}
$$

This is equivalent to the inequality

$$
\left|\psi(z)-\frac{(\mu+1)-(\mu-1) r^{2 n}}{(\mu+1)^{2}-(\mu-1)^{2} r^{2 n}}\right| \leqq \frac{2 r^{n}}{\left[(\mu+1)^{2}-(\mu-1)^{2} r^{2 n}\right]} .
$$

It is easy to show that equality occurs in (2.6) only for functions $P(z)=$ $\left(1-\varepsilon z^{n}\right) /\left(1+\varepsilon z^{n}\right)$ where $|\varepsilon|=1$ and for appropriate values of $z$.

LEMMA 3. If $P(z)$ has a power series expansion of the form

$$
P(z)=1+\sum_{m=1}^{\infty} c_{m k} z^{m k}
$$

then for $|z|=r<1$ and $\mu \geqq 0$,

$$
\left|\frac{z^{2} P^{\prime \prime}(z)}{P(z)+\mu}\right| \leqq \frac{2 k r^{k}}{\left(1-r^{k}\right)^{2}} \frac{(k-1)+(k+1) r^{k}}{(\mu+1)-(\mu-1) r^{k}} .
$$

Proof. Let $P(z)=g\left(z^{k}\right)$. Then $g(z)$ is regular in $E$ and satisfies $g(0)=1$, 
$\operatorname{Re} g(z)>0$ for $z$ in $E$. Let $\zeta$ be a complex number such that $0<|\zeta|<1$. The function

$$
G(z)=g\left(\frac{z+\zeta}{1+\zeta z}\right)=g(\zeta)+\left(1-|\zeta|^{2}\right) g^{\prime}(\zeta) z+\frac{1}{2}\left(1-|\zeta|^{2}\right)\left\{\left(1-|\zeta|^{2}\right) g^{\prime \prime}(\zeta)-2 \bar{\zeta} g^{\prime}(\zeta)\right\} z^{2}
$$

is regular in $E$ and satisfies $\operatorname{Re} G(z)>0$ for $z$ in $E$. Therefore by the CarathéodoryToeplitz theorem, we have

$$
\left|g^{\prime \prime}(\zeta)-\frac{2 \bar{\zeta}}{\left(1-|\zeta|^{2}\right)} g^{\prime}(\zeta)\right| \leqq \frac{4|g(\zeta)|}{\left(1-|\zeta|^{2}\right)^{2}}
$$

This gives

$$
\left|g^{\prime \prime}\left(z^{k}\right)-\frac{2 \bar{z}^{k}}{\left(1-|z|^{2 k}\right)} g^{\prime}\left(z^{k}\right)\right| \leqq \frac{4\left|g\left(z^{k}\right)\right|}{\left(1-|z|^{2 k}\right)^{2}},
$$

for all $z$ in $E$. Using the relation $P(z)=g\left(z^{k}\right)$, we obtain the inequality

$$
\left|z^{2} P^{\prime \prime}(z)-\frac{\left((k-1)+(k+1)|z|^{2 k}\right)}{\left(1-|z|^{2 k}\right)} z P^{\prime}(z)\right| \leqq \frac{4 k^{2}|z|^{2 k}}{\left(1-|z|^{2 k}\right)^{2}}|P(z)| \text {. }
$$

Therefore

$$
\left|\frac{z^{2} P^{\prime \prime}(z)}{P(z)+\mu}\right| \leqq \frac{2 k r^{k}}{\left(1-r^{k}\right)\left[(\mu+1)-(\mu-1) r^{k}\right]} .
$$

From lemma 1, we have for $|z|=r<1$,

$$
\left|\frac{z P^{\prime}(z)}{P(z)+\mu}\right| \leqq \frac{2 k r^{k}}{\left(1-r^{k}\right)\left[(\mu+1)-(\mu-1) r^{k}\right]} .
$$

From lemma 2, we have for $|z|=\mathrm{r}<1$,

$$
\left|\frac{P(z)}{P(z)+\mu}-\frac{(\mu+1)+(\mu-1) r^{2 k}}{(\mu+1)^{2}-(\mu-1)^{2} r^{2 k}}\right| \leqq \frac{2 \mu r^{k}}{\left[(\mu+1)^{2}-(\mu-1)^{2} r^{2 k}\right]} .
$$

The above gives

$$
\left|\frac{P(z)}{P(z)+\mu}\right| \leqq \frac{\left(1+r^{k}\right)}{\left[(\mu+1)-(\mu-1) r^{k}\right]} .
$$

From (2.8), (2.9) and (2.10) we obtain (2.7).

It is easy to show that equality holds in (2.7) only for functions $P(z)=$ $\left(1-\varepsilon z^{k}\right) /\left(1+\varepsilon z^{k}\right)$ where $|\varepsilon|=1$ and for appropriate values of $z$.

THEOREM 1. Suppose that $f(z)=z+a_{k+1} z^{k+1}+a_{2 k+1} z^{2 k+1}+\cdots$ is regular in $E$ and satisfies $|(f(z) \mid z)-\alpha|<\alpha\left(\alpha>\frac{1}{2}\right)$ for $z$ in E. Then $f(z)$ maps $|z|<r_{\alpha}$ onto 
a convex domain where $r_{\alpha}$ is the smallest positive root of the equation

$$
\begin{gathered}
x^{2}-\alpha\left((2-\alpha)+(2 \alpha-1)\left(2 k+k^{2}\right)\right) r^{k}+(1-\alpha)\left((1+\alpha)+(2 \alpha-1)\left(2 k-k^{2}\right)\right) r^{2 k} \\
-(1-\alpha)^{2} r^{3 k}=0 .
\end{gathered}
$$

This result is sharp in the sense that the number $r_{\alpha}$ cannot be replaced by any larger one.

ProOF. Let

$$
\psi(z)=1-\frac{1}{\alpha} \frac{f(z)}{z},
$$

then $\psi(z)$ is regular in $E,|\psi(z)|<1$ for $z$ in $E$ and $\psi(0)=1-(1 / \alpha)$. Let

$$
F(z)=\frac{\psi(z)-\psi(0)}{1-\psi(0) \psi(z)},
$$

then $F(z)$ is regular in $E,|F(z)|<1$ for $z$ in $E$ and $F(0)=0$. Also $F(z)$ has a power series expansion of the form $F(z)=b_{k} z^{k}+b_{2 k} z^{2 k}+\cdots$. Such a function $F(z)$ can be represented as $[7$, p. 169]

$$
F(z)=\frac{P(z)-1}{P(z)+1}
$$

Evidently $P(z)$ has a power series expansion of the form $P(z)=1+c_{k} z^{k}+c_{2 k} z^{2 k}$ $+\cdots$. From (2.11), (2.12) and (2.13) we have

$$
f(z)=\frac{2 \alpha z}{1+(2 \alpha-1) P(z)} .
$$

The representation (2.14) yields the relation

$$
1+\frac{z f^{\prime \prime}(z)}{f^{\prime}(z)}=1-\frac{2 z P^{\prime}(z)}{P(z)+(1 /(2 \alpha-1))}-\frac{z^{2} P^{\prime \prime}(z)}{(1 /(2 \alpha-1))+P(z)-z P^{\prime}(z)} .
$$

From lemmas 1 and 3 we have for $|z|=r, 0 \leqq r<1$,

$$
\left|\frac{z P^{\prime}(z)}{P(z)+(1 /(2 \alpha-1))}\right| \leqq \frac{(2 \alpha-1) k r^{k}}{\alpha-r^{k}-(\alpha-1) r^{2 k}}=\frac{(2 \alpha-1) k r^{k}}{\left(1-r^{k}\right)\left(\alpha-(1-\alpha) r^{k}\right)},
$$

and

$$
\left|\frac{z^{2} P^{\prime \prime}(z)}{P(z)+(1 /(2 \alpha-1))}\right| \leqq \frac{(2 \alpha-1) k r^{k}\left((k-1)+(k+1) r^{k}\right)}{\left(1-r^{k}\right)^{2}\left(\alpha-(1-\alpha) r^{k}\right)}
$$

Let

$$
R=\left[\frac{(1+(2 \alpha-1) k)-\left\{(2 \alpha-1)\left((2 \alpha-1)\left(1+k^{2}\right)+2 k\right)\right\}^{\frac{1}{2}}}{2(1-\alpha)}\right]^{1 / k}
$$


From (2.16) and (2.17) we have for $|z|=r, 0 \leqq r<R$,

$$
\left|\frac{z^{2} P^{\prime \prime}(z)}{(1 /(2 \alpha-1))+P(z)-z P^{\prime}(z)}\right| \leqq \frac{k(2 \alpha-1) r^{k}\left((k+1) r^{k}+(k-1)\right)}{\left(1-r^{k}\right)\left((1-\alpha) r^{2 k}-(1+k(2 \alpha-1)) r^{k}+\alpha\right)} \text {. }
$$

From (2.15), (2.16) and (2.19) we have for $|z|=r, 0 \leqq r<R$,

$$
\begin{aligned}
\operatorname{Re}\left(1+\frac{z f^{\prime \prime}(z)}{f^{\prime}(z)}\right) \geqq & 1-\frac{2(2 \alpha-1) k r^{k}}{\left(1-r^{k}\right)\left(\alpha-(1-\alpha) r^{k}\right)} \\
& -\frac{k(2 \alpha-1) r^{k}\left((k+1) r^{k}+(k-1)\right)}{\left(1-r^{k}\right)\left((1-\alpha) r^{2 k}-(1+k(2 \alpha-1)) r^{k}+\alpha\right)} \\
= & \frac{p(r)}{\left(\alpha-(1-\alpha) r^{k}\right)\left((1-\alpha) r^{2 k}-(1+k(2 \alpha-1)) r^{k}+\alpha\right)},
\end{aligned}
$$

where

$$
\begin{aligned}
p(r)=\alpha^{2}-\alpha\{(2-\alpha)+ & \left.(2 \alpha-1)\left(2 k+k^{2}\right)\right\} r^{k} \\
& +(1-\alpha)\left\{(1+\alpha)+(2 \alpha-1)\left(2 k-k^{2}\right)\right\} r^{2 k}-(1-\alpha)^{2} r^{3 k} .
\end{aligned}
$$

The condition $\operatorname{Re}\left(1+\left(z f^{\prime \prime}(z) / f^{\prime}(z)\right)\right)>0$ for $|z|<r$ is necessary and sufficient for $f(z)$ to map $|z|<r$ onto a convex domain. From the above estimate, we see that this condition is satisfied in $|z|<\min \left(r_{\alpha}, R\right)$ where $r_{\alpha}$ is the smallest positive root of the equation $p(r)=0$. Writing $p(r)$ as

$$
\begin{aligned}
p(r)= & \left(\alpha-(1-\alpha) r^{k}\right)\left((1-\alpha) r^{2 k}-\left(1+k(2 \alpha-1) r^{k}+\alpha\right)\right. \\
& +k(2 \alpha-1) r^{k}\left((1-k)(1-\alpha) r^{k}-\alpha(1+k)\right)
\end{aligned}
$$

and using (2.18) it is easily verified that $r_{\alpha}<R$. Hence $f(z)$ is convex in $|z|<r_{\alpha}$.

The function

$$
f_{\alpha}(z)=\frac{\alpha\left(z+z^{k+1}\right)}{\alpha+(1-\alpha) z^{k}}=z+\left(2-\frac{1}{\alpha}\right) z^{k+1}+\cdots
$$

satisfies the hypothesis of the above theorem but is not convex in $|z|<r$ with $r \geqq r_{\alpha}$.

Letting $\alpha$ tend to infinity and putting $k=1$ in theorem 1, we get the result of Reade, Ogawa and Sakaguchi [8].

THEOREM 2. Suppose that $f(z)=z+a_{n} z^{n}+a_{n+1} z^{n+1}+\cdots$ is regular in $E$ and satisfies $|(f(z) / z)-\alpha|<\alpha\left(\alpha>\frac{1}{2}\right)$ for $z$ in $E$. Then $f(z)$ maps $|z|<r_{\alpha}$ onto a univalent and starlike domain where

$$
r_{\alpha}=\left\{\begin{array}{l}
{[\{2(1-\alpha)+(2 \alpha-1) n} \\
\left.\left.-\left((2(1-\alpha)+(2 \alpha-1) n)^{2}+4 \alpha(\alpha-1)\right)^{\frac{1}{2}}\right\} / 2(1-\alpha)\right]^{1 /(n-1)} \\
2^{-1 /(n-1)} \text { if } \alpha=1 .
\end{array}\right.
$$

This result is sharp. 
Proof. Proceeding as in theorem 1, we have

$$
f(z)=\frac{2 \alpha z}{1+(2 \alpha-1) P(z)},
$$

where $P(z)$ has a power series expansion of the form $P(z)=1+c_{n-1} z^{n-1}+\cdots$. The representation (2.21) yields

$$
\frac{z f^{\prime}(z)}{f(z)}=1-\frac{(2 \alpha-1) z P^{\prime}(z)}{1+(2 \alpha-1) P(z)}
$$

Taking real parts on both sides of (2.23) and using lemma 1 we have for $|z|=$ $r, 0 \leqq r<1$,

$$
\operatorname{Re}\left(\frac{z f^{\prime}(z)}{f(z)}\right) \geqq \frac{\alpha-(2(1-\alpha)+(2 \alpha-1) n) r^{n-1}+(1-\alpha) r^{2(n-1)}}{\alpha-r^{n-1}-(\alpha-1) r^{2(n-1)}} .
$$

A necessary and sufficient condition for $f(z)$ to map $|z|<r$ onto a univalent and starlike domain is that $\operatorname{Re}\left(z f^{\prime}(z) / f(z)\right)>0$ for $|z|<r$. From the above estimate we see that this condition is satisfied for $|z|<r_{\alpha}$ where $r_{\alpha}$ is given by (2.21).

The function

$$
f_{\alpha}(z)=\frac{\alpha\left(z+z^{n}\right)}{\alpha+(1-\alpha) z^{n-1}}=z+\left(2-\frac{1}{\alpha}\right) z^{n}+\cdots
$$

satisfies the hypothesis of the above theorem but is not univalent in $|z|<r$ with $r \geqq r_{\alpha}$ for $f_{\alpha}^{\prime}(z)$ vanishes at $z=r_{\alpha} \exp (i \pi /(n-1))$.

Let $F(z)=z f^{\prime}(z)$. If $F(z)$ be starlike with respect to the origin in $|z|<r$ then $f(z)$ is convex in $|z|<r$ [7, p. 223]. Also if $f(z)$ satisfies $\left|f^{\prime}(z)-\alpha\right|<\alpha$ for $z$ in $E$, then $F(z)$ satisfies $|(F(z) / z)-\alpha|<\alpha$ for $z$ in $E$. Therefore we arrive at

COROLlaRY 2.1. Suppose that $f(z)=z+a_{n} z^{n}+a_{n+1} z^{n+1}+\cdots$ is regular in $E$ and satisfies $\left|f^{\prime}(z)-\alpha\right|<\alpha\left(\alpha>\frac{1}{2}\right)$ for $z$ in $E$. Then $f(z)$ maps $|z|<r_{\alpha}$ onto a convex domain where $r_{\alpha}$ is given by (2.21). This result is sharp, the extremal function being

$$
f_{\alpha}(z)=\int_{0}^{z} \frac{\alpha\left(1+z^{n-1}\right)}{\alpha+(1-\alpha) z^{n-1}} d z
$$

Letting $\alpha$ tend to infinity and putting $n=2$ in theorem 2 and corollary 2.1 , we get the results of MacGregor [4].

THEOREM 3. Suppose that $f(z)$ is regular in $E$ and satisfies $f(0)=0, f^{\prime}(0)=1$ and $\left|\left(f(z) /\left(z+a z^{2}\right)\right)-\alpha\right|<\alpha\left(|a| \leqq \mid, \alpha>\frac{1}{2}\right)$ for $z$ in E. Then $f(z)$ maps $|z|<r_{\alpha}(|a|)$ onto a univalent and starlike domain where $r_{\alpha}(|a|)$ is the smallest positive root of 
the equation

$$
\alpha-2 \alpha(1+|a|) r+((1-\alpha)+(2 \alpha+1)|a|) r^{2}-2(1-\alpha)|a| r^{3}=0 .
$$

This result is sharp, the extremal function being

$$
f(z)=\frac{1+z e^{i \gamma}}{1+\left(\frac{1}{\alpha}-1\right) z e^{i \gamma}}\left(z+a z^{2}\right), \quad(\gamma=\arg a,|a| \leqq 1) .
$$

The number $r_{\alpha}(|a|)$ decreases monotonically as $|a|$ increases from 0 to 1 .

The case $a=0$ and $\alpha=1$ is Theorem 1 of [6].

The proof of the above theorem is similar to that of theorem 2 and is therefore omitted.

THEOREM 4 (A) Suppose that $f(z)$ is regular in $E$ and satisfies $f(0)=0$, $f^{\prime}(0)=1$ and $\operatorname{Re}\left(f(z) /\left(z+a z^{2}\right)\right)>0(|a| \leqq 1)$ for $z$ in $E$. Then $f(z)$ maps $|z|<r$ $(|a|)$ onto a convex domain where $r(|a|)$ is the smallest positive root of the equation

$$
1-(5+4|a|) r+(6|a|-3) r^{2}+(10|a|-1) r^{3}+4|a| r^{4}=0 .
$$

(B) Suppose that $f(z)$ is regular in $E$ and satisfies $f(0)=0, f^{\prime}(0)=1$ and $\left|\left(f(z) \mid\left(z+a z^{2}\right)\right)-1\right|<1(|a| \leqq 1)$ for $z$ in $E$. Then $f(z)$ maps $|z|<r_{1}(|a|)$ onto a convex domain where

$$
r_{1}(|a|)=\left\{\begin{array}{l}
\frac{1}{4} \quad \text { if } a=0, \\
{\left[2(1+|a|)-\left\{4\left(1+|a|^{2}\right)-|a|\right\}^{\frac{1}{2}}\right] /(9|a|) \quad \text { if } a \neq 0 .}
\end{array}\right.
$$

The above estimates are sharp and decrease monotonically as $|a|$ increases from 0 to 1.

Proof OF Theorem 4(A). Let $g(z)=z+a z^{2}$. It is easy to see that for $|z|=r$, $0 \leqq r<1 /(2|a|)$

$$
\left|\frac{z g^{\prime \prime}(z)}{g(z)}\right| \leqq \frac{2|a| r}{1-|a| r}, \quad\left|\frac{z g^{\prime}(z)}{g(z)}\right| \geqq \frac{1-2|a| r}{1-|a| r} .
$$

Let

$$
\frac{f(z)}{g(z)}=\frac{1}{P(z)} .
$$

The representation (3.2) yields the relation

$$
1+\frac{z f^{\prime \prime}(z)}{f^{\prime}(z)}=1-\frac{2 z P^{\prime}(z)}{P(z)}-\frac{\frac{z^{2} P^{\prime \prime}(z)}{P(z)}-\frac{z^{2} g^{\prime \prime}(z)}{g(z)}}{\frac{z g^{\prime}(z)}{g(z)}-\frac{z P^{\prime}(z)}{P(z)}} .
$$


Using (3.1) and lemma 1, we have for $|z|=r, 0 \leqq r<1 /(2|a|)$,

$$
\begin{aligned}
\left|\frac{z g^{\prime}(z)}{g(z)}-\frac{z P^{\prime}(z)}{P(z)}\right| \geqq \frac{1-2|a| r}{1-|a| r}-\frac{2 r}{1-r^{2}} & \\
& =\frac{1-2(1+|a|) r+(2|a|-1) r^{2}+2|a| r^{3}}{\left(1-r^{2}\right)(1-|a| r)} .
\end{aligned}
$$

Let $r_{0}$ be the smallest positive root of the equation

$$
\psi(r) \equiv 1-2(1+|a|) r+(2|a|-1) r^{2}+2|a| r^{3}=0 .
$$

It is easy to verify that $r_{0}<1 /(2|a|)$. Therefore using lemmas 1 and 3 and the inequalities (3.1) and (3.4), we have for $|z|=r, 0 \leqq r<r_{0}$,

$$
\operatorname{Re}\left(1+\frac{z f^{\prime \prime}(z)}{f^{\prime}(z)}\right) \geqq \frac{1-(5+4|a|) r+(6|a|-3) r^{2}+(10|a|-1) r^{3}+4|a| r^{4}}{(1+r)\left[1-2(1+|a|) r+(2|a|-1) r^{2}+2|a| r^{3}\right]} .
$$

Let $r(|a|)$ be the smallest positive root of the equation

$$
\chi(r) \equiv 1-(5+4|a|) r+(6|a|-3) r^{2}+(10|a|-1) r^{3}+4|a| r^{4}=0 .
$$

It is easy to verify that $0<r(|a|)<\frac{1}{4}$. Also $\psi(r)$ is monotonically decreasing for $0 \leqq r \leqq \frac{1}{4}$ and $\psi\left(\frac{1}{4}\right)$ is positive. Therefore $r_{0}>\frac{1}{4}$. Thus we see that $\operatorname{Re}\left(1+\left(z f^{\prime \prime}(z)\right)\right.$ $\left.\left.f^{\prime}(z)\right)\right)>0$ for $|z|=r<r(|a|)$, which implies that $f(z)$ maps $|z|<r(|a|)$ onto a convex domain.

The above estimate is sharp because the function

$$
f(z)=\frac{1+z e^{i \gamma}}{1-z e^{i \gamma}}\left(z+a z^{2}\right) \quad(\gamma=\operatorname{arga},|a| \leqq 1)
$$

satisfies the hypothesis of the above theorem but is not convex in $|z|<r$ with $r \geqq r(|a|)$.

It is easy to verify that $r(|a|)$ decreases monotonically as $|a|$ increases from 0 to 1 .

Theorem $4(\mathrm{~B})$ can be proved in the same manner as theorem $4(\mathrm{~A})$. The extremal function in this case is

$$
f(z)=\left(1+e^{i \gamma} z\right)\left(z+a z^{2}\right) \quad(\gamma=\operatorname{arga},|a| \leqq 1) .
$$

TYPICALLY-REAL FUNCTIONS The function $f(z)=z+a_{2} z^{2}+\cdots$, regular in $E$ is called typically-real in $E$ if it is real on the diameter $-1<z<1$ and if at other points of the circle $E, \operatorname{Im}(f(z)) \cdot \operatorname{Im}(z)>0$, see [11]. The radius of starlikeness of this class is $(\sqrt{ } 2-1)$, see [2]. We find below the radius of starlikeness of a subclass of the class of typically-real functions. 
THEOREM 5. Suppose that $f(z)$ is regular and real on the real axis in $E$ and satisfies the conditions $f(0)=0, f^{\prime}(0)=1, f^{\prime \prime}(0)=0$ and

$$
\left|\frac{\left(1-z^{2}\right)}{z} f(z)-\alpha\right|<\alpha \quad\left(\alpha>\frac{1}{2}\right),
$$

for all $z$ in $E$. Then $f(z)$ is univalent and starlike in $|z|<r_{\alpha}$ where $r_{\alpha}$ is the smallest positive root of the equation

$$
\alpha-(5 \alpha-1) r^{2}-(5 \alpha-4) r^{4}+(\alpha-1) r^{6}=0 .
$$

This estimate is sharp, the extremal function being

$$
f_{\alpha}(z)=\frac{\alpha z\left(1+z^{2}\right)}{\left(1-z^{2}\right)\left(\alpha+(1-\alpha) z^{2}\right)}=z+\left(3-\frac{1}{\alpha}\right) z^{3}+\cdots .
$$

Remark. A necessary and sufficient condition that $f(z)$ be typically real in $E$ is that $\operatorname{Re}\left\{\left(1-z^{1}\right) / z f(z)\right\}>0$ and $1-z^{2} / z f(z)$ is real on the real axis for $z$ in $E$, [10]. Evidently the functions which satisfy the hypothesis of the above theorem satisfy this condition and therefore form a subclass of the class of typically real functions.

Proof Of Theorem 5. Proceeding as in theorem 1 we have

$$
f(z)=\frac{2 \alpha z}{\left(1-z^{2}\right)(1+(2 \alpha-1) P(z))},
$$

where $P(z)$ has a power series expansion of the form $P(z)=1+c_{2} z^{2}+\cdots$. The representation (4.3) yields the relation

$$
\frac{z f^{\prime}(z)}{f(z)}=\frac{1+z^{2}}{1-z^{2}}-\frac{(2 \alpha-1) z P^{\prime}(z)}{(1+(2 \alpha-1) P(z))} .
$$

From lemma 1, we have for $|z|=r<1$,

$$
\left|\frac{z P^{\prime}(z)}{(1 /(2 \alpha-1))+P(z)}\right| \leqq \frac{2(2 \alpha-1) r^{2}}{\left(1-r^{2}\right)\left(\alpha-(1-\alpha) r^{2}\right)} .
$$

From (4.4) and (4.5) we have for $|z|=r<1$,

$\operatorname{Re}\left(\frac{z f^{\prime}(z)}{f(z)}\right) \geqq \frac{1-r^{2}}{1+r^{2}}-\frac{2(2 \alpha-1) r^{2}}{\left(1-r^{2}\right)\left(\alpha-(1-\alpha) r^{2}\right)}$

$$
=\frac{\alpha-(5 \alpha-1) r^{2}-(5 \alpha-4) r^{4}+(\alpha-1) r^{6}}{\left(1-r^{4}\right)\left(\alpha-(1-\alpha) r^{2}\right)} .
$$

Thus we see that $\operatorname{Re}\left(\left(z f^{\prime}(z)\right) / f(z)\right)>0$ in $|z|<r_{\alpha}$ where $r_{\alpha}$ is the smallest positive root of (4.2). 
If $f(z)$ be typically-real, then $F(z)=z f^{\prime}(z)$ is real on the real axis and convex in the direction of the imaginary axis [10]. Therefore we arrive at

COROLlaRY 5.1. Suppose that $f(z)$ is regular in $E$ and satisfies the conditions $t(0)=0, f^{\prime}(0)=1, f^{\prime \prime}(0)=0$ and

$$
\left|\left(1-z^{2}\right) f^{\prime}(z)-\alpha\right|<\alpha \quad\left(\alpha>\frac{1}{2}\right),
$$

for $|z|<1$. Then $f(z)$ is convex in $|z|<r_{\alpha}$ where $r_{\alpha}$ is the smallest positive root of fhe equation (5.2). This result is sharp, the extremal function being

$$
f_{\alpha}(z)=\int_{0}^{z} \frac{\alpha\left(1+z^{2}\right)}{\left(1-z^{2}\right)\left(\alpha+(1-\alpha) z^{2}\right)} d z
$$

\section{Acknowledgements}

My thanks are due to Professor Vikramaditya Singh for his helpful guidance in the preparation of this paper.

\section{References}

[1] G. M. Goluzin, Some estimations of derivatives of bounded functions, Rec. Math. Mat. Sbornik N.S. 16 (58) (1945), 295-306.

[2] W. E. Kirwan, Extremal problems for the typicallyreal functions, Amer. J. Math. 88 (1966).

[3] R. J. Libera, 'Some radius of convexity problems', Duke Math. J. 31 (1964), 143-158.

[4] T. H. MacGregor, 'Functions whose derivative has a positive real part', Trans. Amer. Math. Soc. 104 (1962), 532-537.

[5] T. H. MacGregor, 'The radius of univalence of certain analytic functions', Proc. Amer.Math. Soc. 14 (1963), 514-520.

[6] T. H. MacGregor, 'The radius of univalence of certain analytic functions II', Proc. Amer. Math. Soc. 14 (1963), 521-524.

[7] Z. Nehari, Conformal Mapping, (McGraw-Hill, New York, 1952).

[8] M. O. Reade, S. Ogawa and K. Sakaguchi, 'The radius of convexity for a certain class of analytic functions', J. Nara Gakugei Univ. (Nat.) 13 (1965), 1-3.

[9] M. O. Reade, 'On close-to-convex univalent functions', Mich. Math. J., 3 (1955-1956), $59-62$.

[10] M. S. Robertson, 'On the theory of univalent functions, Annals of Mathematics', 37 (1936) $374-408$.

[11] W. Rogosinski, 'Uber positive harmonische Entwicklungen und typisch-reele Potenzreihen, Mathematische Zeitschrift, 35 (1932), 93-121.

Department of Mathematics

Punjabi University

Patiala, India 